\title{
An Efficient Compact Flexible EM Energy Harvester
}

\author{
Khaled A. Aljaloud, Student Member, IEEE, Kin-Fai. Tong, Senior Member, IEEE \\ Electronic and Electrical Engineering Department \\ Roberts Building, University College London \\ Torrington Place, London \\ WC1E 7JE \\ Khaled.aljaloud.15@ucl.ac.uk
}

\begin{abstract}
In this paper, we present a compact flexible electromagnetic (EM) energy harvester systems using a small loop resonator. They are designed and fabricated on a flexible substrate, and their performance are verified through measurement for both flat and curved configurations. The efficiency is investigated with respect to power density. It is observed that the proposed systems can achieve high conversion efficiency for low input power while at the same time occupying a compact footprint areas. In addition, the output DC power by harvester system using multi-resonator array is compered to a system with a single resonator. The measurement results demonstrate that an enhancement in the performance of up to 4 times are achieved.

Index Terms-electromagnetic energy harvesting and wireless power transmission.
\end{abstract}

\section{INTRODUCTION}

The electromagnetic (EM) energy harvester is a combination of receiving antenna, or EM collector, and rectifier circuit. It has empowered various applications in numerous areas since their essential objective is to harvest and reuse the surrounding RF power. Flexible electronic devices have received a considerable intention during the past decade where they can be used in many potential applications such as wearable medical device applications. Rigid EM energy harvester systems have been already proposed in the energy harvesting literature. Conformal EM harvester, on the other hand, has few examples [1] [3]. Furthermore, the majority of those systems require high input power or large layout area to obtain the peak efficiency. Therefore, increasing the capability of EM energy harvester for low input power while at the same time occupying a smaller footprint area is in high demand.

In this letter, we present a compact flexible EM energy harvester systems for capturing low-power RF energy. The system with single collector is not adequate to harvest and produce sufficient DC power; therefore, we propose an EM energy harvester using multi-resonator array: 2 serial and 2 parallel (S2P2). We show that when an EM energy harvester employing a multi-resonator array, the DC power out of the system experiences a notable improvement over the system with the use of a single resonator for the same excitation and power levels.

\section{Design AND Result}

\section{A. EM Energy Harvester Design}

An unit cell of the proposed EM energy harvester is shown in Fig. 1(a). It is composed of a small loop resonator, a series diode half wave rectifier circuit. A full wave simulator (CST) was used to optimize the loop resonator to operate at $2.45 \mathrm{GHz}$. The length of each side of the antenna is $\mathrm{L}=$ $10.8 \mathrm{~mm}$ and the width of the line is $\mathrm{W}=0.55 \mathrm{~mm}(4 \mathrm{~L}$ $\approx 0.4 \lambda_{o}$ ). The rectifier circuit consists of a schottky diode connected in series to one of antenna feed terminals, a $27 p F$ capacitor shunted across the coplanar waveguide (CPW) lines to reduce the ripple level, and an optimized $560 \Omega$ resistive load connected to the output of the system for receiving the DC power. Schottky diodes from Skyworks SMS7630 is used because of the low barrier height $(0.69 \mathrm{eV})$, which results in good sensitivity, and the low parasitic capacitance that makes the diode a superior detector at high frequencies. The EM harvester is fabricated on flexible substrate (RO3003) with $0.13 \mathrm{~mm}$ thickness $35 \mu \mathrm{m}$ copper cladding as displayed in Fig. 1(b).

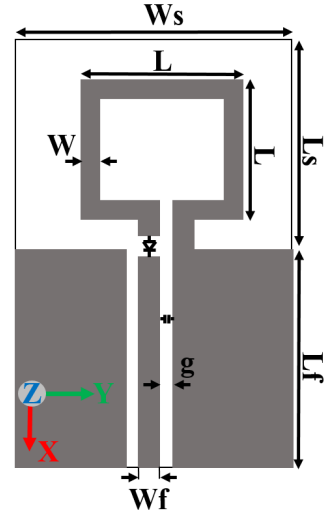

(a)

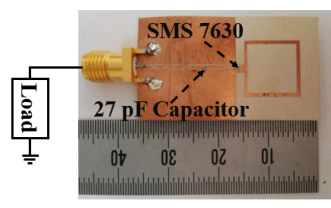

(b)

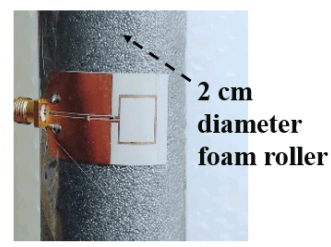

(c)
Fig. 1. (a) Configuration of the proposed harvester, and the parameters are: $W_{s}=20 \mathrm{~mm}, L_{s}=18 \mathrm{~mm}, L_{f}=18 \mathrm{~mm}, L=10.8 \mathrm{~mm}$, $W=0.55 \mathrm{~mm}, W_{f}=0.5 \mathrm{~mm}, g=0.3 \mathrm{~mm}$. Fabricated prototypes: (b) Flat structure. (c) Curved structure.

The number of energy collectors in the EM energy harvesting system is critical to maximize the captured power. 
Therefore, having an array of EM collector is in demand for improve the performance in such a system. An energy harvesting system was designed using loop resonator array connected to a simple series half wave rectifier circuit. The elements of the array connected in series and parallel. The horizontal and vertical spacing between any two adjacent elements are 1.4 $\mathrm{mm}(0.0114 \mathrm{o})$ and $5.2 \mathrm{~mm}(0.0426 \mathrm{o})$, respectively. The system was fabricated on flexible substrate (RO3003) as shown in Fig. 2(a).

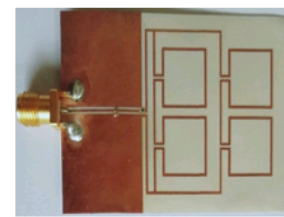

(a)

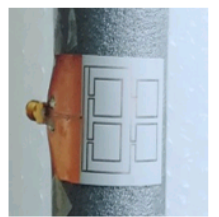

(b)
Fig. 2. Fabricated prototypes of S2P2: (a) Flat structure and (b) Curved structure.

\section{B. Experimental Result}

The performance of the EM energy harvester systems were experimentally verified. A RF signal generator has been connected to a $8 \mathrm{~dB}$ gain horn antenna transmitter placed at a distance of $1.1 \mathrm{~m}$ from the EM energy harvester to satisfy the far field condition. The power conversion efficiency is the ratio between the output DC power and the input radiated power, which is obtained from the footprint area of the resonator and the incident power densities on the EM energy harvester.

By utilizing the Friss transmission equation for free-space wave propagation, power conversion efficiency $\left(\eta_{P C E}\right)$ was measured as a function of power density for both proposed configurations. The comparison between these two systems is shown in Fig. 4, which indicates that: By expanding the footprint and bringing the elements closer to each other, an improvement of $7 \%$ in conversion efficiency was achieved with $\mathrm{S} 2 \mathrm{P} 2$ compared with only one element of loop resonator. This is because of the constructive coupling between collectors.

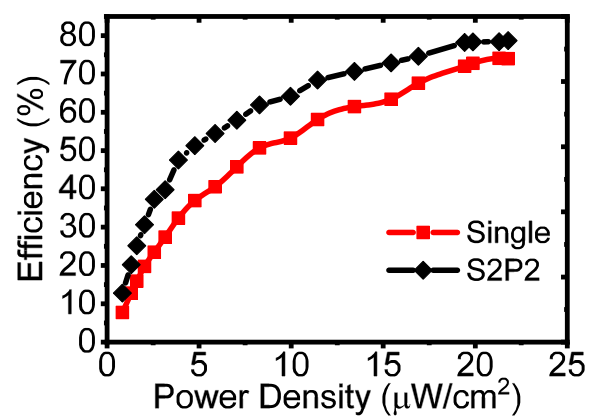

Fig. 3. Measurement efficiency of the fabricated systems with respect to the incident power density.

At $20 \mu \mathrm{W} / \mathrm{cm}^{2}$ power density, the DC power out of these systems was measured over an optimized $560 \Omega$ resistive load as a function of frequency (Fig. 4), and the performance of these systems was also tested when they were curved and mounted on $2 \mathrm{~cm}$ diameter foam roller (Fig. 1(c) and Fig.2(b)) to ensure that they maintain their operating frequency when they are bent on a curved structure. From these results shown in Fig.4, we notice a number of things: (1) The power collected by $\mathrm{S} 2 \mathrm{P} 2$ is four times the power collected by an EM energy harvester with a single resonator. (2) Peak DC power out of these systems were recorded at the operating frequency. Since the EM energy harvester systems were designed to harvest EM waves at $2.45 \mathrm{GHz}$ with reasonable impedance matching with the rectifier circuit, the relationship between output DC power and frequency was found to be in good agreement with design targets. (3) The operating frequencies for both flat and curved configurations are the same. Furthermore, peak output DC power at the operating frequency maintain unchanged.

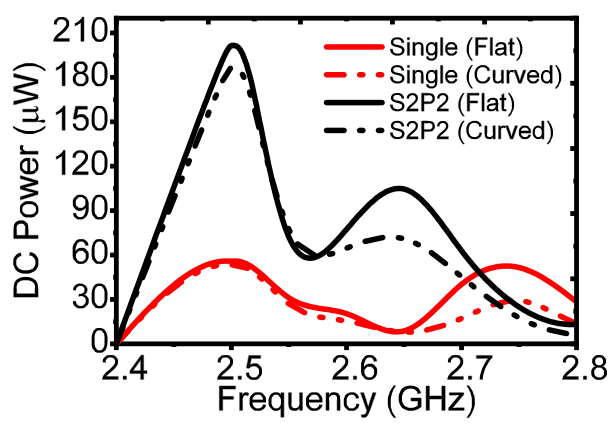

Fig. 4. Measured DC output power of the fabricated systems as a function of the frequency, when the power density is $20 \mu \mathrm{W} / \mathrm{cm}^{2}$, for different configurations: flat and curved.

\section{CONCLUSIONS}

A single compact EM energy harvester based on loop resonator was designed, implemented on flexible substrate, and then verified. A $72 \%$ conversion efficiency was achieved with power density as low as of $20 \mu \mathrm{W} / \mathrm{cm}^{2}$. To improve the performance, EM energy harvester based on multi-resonator array was proposed. A comparison between the systems was demonstrated. It is observed that the system using multiresonator array can improve collected power by up to 4 times, compared to a system with single resonator.

The EM energy harvester systems presented here achieve high efficiencies at low power density $\left(20 \mu \mathrm{W} / \mathrm{cm}^{2}\right)$ have many applications when the radiating power is low or the source of radiation is far away. Moreover, the flexibility of the proposed systems can be used for conformal applications.

\section{REFERENCES}

[1] S.-E. Adami et al., "A Flexible 2.45-GHz Power Harvesting Wristband With Net System Output From -24.3 dBm of RF Power," IEEE Trans. Microw. Theory Techn., pp. 1-16, 2017.

[2] V. Palazzi et al., "Performance analysis of a ultra-compact low-power rectenna in paper substrate for RF energy harvesting," IEEE Topical Conf. Wireless Sensors Sensor Netw. (WiSNet), Jan. 2017, pp. 6568.

[3] A. Collado and A. Georgiadis, "Conformal Hybrid Solar and Electromagnetic (EM) Energy Harvesting Rectenna," IEEE Trans. Circuits Syst. I, Reg. Papers, vol. 60, no. 8, pp. 2225-2234, 2013. 